\title{
Automatic Underground Cylindrical Multi-Levels Car Parking Secured Management System
}

\author{
Elsanosy M. Elamin ${ }^{1}$, Abdelrasoul Jabar Alzubaidi ${ }^{2}$
}

${ }^{1}$ Electrical Engineering Department, Faculty of Engineering, University of Kordofan, Sudan ${ }^{2}$ Electronics Engineering School, Sudan University of Science and Technology, Khartoum, Sudan

\begin{abstract}
As the all technologies that relevant to the human are developing rapidly, it is found that the number of new models of cars are also increased with high technology. Due to the increasing growth of cars and investment in all aspect of life, the parking areas are definitely start to decrease to yield a significant problem. This work deals with the relevant problem as smart design to take part in finding better solution. The paper is divided into hardware and software and the integration of them to monitor and control the process automatically. The crucial feature that appended to the design is the security aspect to enable the user to use the system with high reliability. The design exploited PC instead of microcontroller to facilitate the human machine interface. The design also equipped with printer to provide the user with security code when calling back or retrieving the car. The overall work shows the security, reliability, and high revenue. It is applicable in many different field such as hotels, shopping malls, and etc.
\end{abstract}

Keywords: car parking, stepper motor, hydraulic mechanism, parking room, IR sensors.

\section{INTRODUCTION}

The recent technologies nowadays are tending to minimize the size, power consumption, and operational time so as to save both overall time and parking area as well as making use of technologies integration. The micro-electronic technology drives most of the electronic and control designers to meet the current situation challenges [1], [2]. This paper deals directly with the controlling of the parking process of the car in cylindrical multi-levels parking rooms to minimize the parking area and to raise up the parking revenue where the space in buildings is the competitive factor. In addition, the parking time always assist in reducing the overall parking management processes. The fundamental concept of the work is relying on how to realize the empty closer rooms in the cylindrical parking to be occupied in order to save time in both storing and retrieving the car securely. To satisfy such condition, the controlling software program is performed via PC to monitor and control the parking management processes. Sensors, cameras, stepper motors, printer, pallet, and hydraulic mechanism are used to facilitate the parking management process and introduces a cost effective solution.

\section{The System COMPONENTS}

\section{System Hardware}

The hardware of the system is consisting of vital parts: entrance gate, IR and weight sensors, PC, relays, motors, two hydraulic mechanisms, and parking rooms as shown in figure 1.

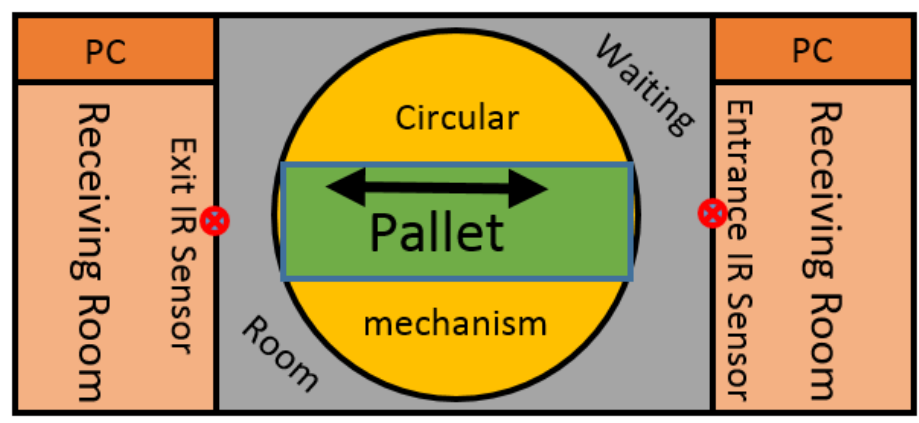

Figure1. The hardware system parts 


\subsection{Entrance Gate}

It is the main gate that enable the car to step forward to be taken to the hydraulic lift so as to be parked inside the room. This gate is connected to the main software program to decide whether to allow the car to enter to the circular holder to be stored/parking in the room or not according to the parking capacity or congestion.

\subsection{IR and weight sensors}

The system contains about 27 sensors. 24 of them are used to check the parking room whether it is empty or occupied and two for the entrance and exit gates to check if there is/are empty room(s) or not to allow the incoming car enter the system or block it. In addition, there is weight sensor to ensure that the parked object it is a car. The sensing process is done through infrared transmitters and receivers. The infrared transmitter is mounted on one side of the parking room and the receiver in the other opposite side of the transmitter. When a car reached the parking room, the infrared beam is blocked and the receiver is devoid of infrared. message is sent to the PC to declare that the car is parked. In addition, there is a weight sensor that attached to the hydraulic lift mechanism to insure that the serviced body has a weight of car. The car weight is entered to the system not to exceed the hydraulic capacity for better operation and safety [3].

\subsection{Controlling PC}

The controlling software program is stored in the PC for better performance. The PC is well interfaced to the all system components to carry out all the monitoring and controlling process

\subsection{Relays}

The devices of the system components that used in design are all of heavy current devices. In order to be directly controlled, the relays, which are a part of control circuit, are exploited as interface circuits to help in driving both high torque motors and hydraulic mechanisms. [1]

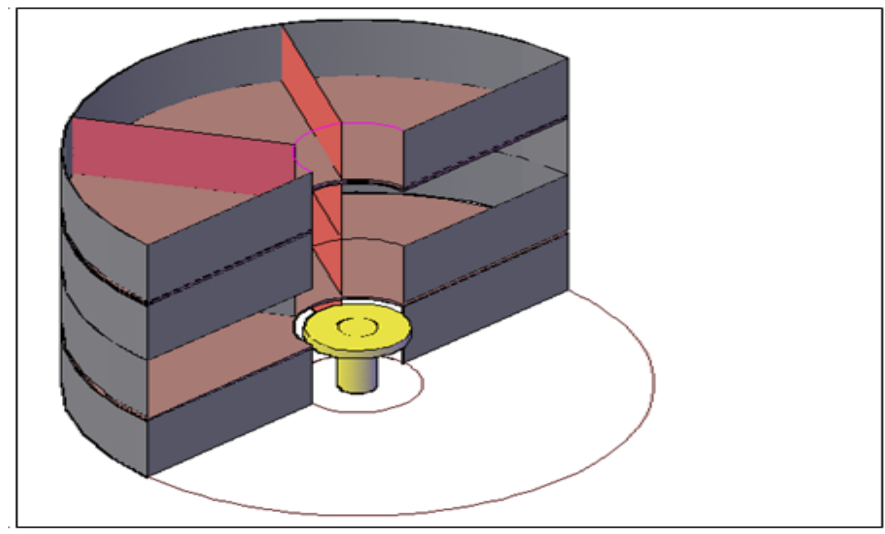

Figure2. Illustrate the parking room

\subsection{Hydraulic mechanisms}

This mechanism composed of Hydraulic lift and Pallet. The lift mechanism consists of three moving parts. The first part is hydraulic lift which has a circular head that can move circularly in order to choose the reasonable empty room(s) in a cylindrical shape. The circular head also consists of pallet that can move horizontally inward/outward so as to put the car inside the room or to pull it out when retrieving the car.

\subsection{Parking Room}

The cylindrical car parking building is composed of four floors with six parking rooms for each to form about 24 car parking rooms as stated in figure 2 that shows the cross-sectional view of the system. The parking rooms is equipped with sensors to reveal whether all or some of it are occupied or empty. In addition, the camera is also used to monitor the parking management process and in same time to insure the sensors performance. 
The block diagram of system components is shown in figure 3 below.

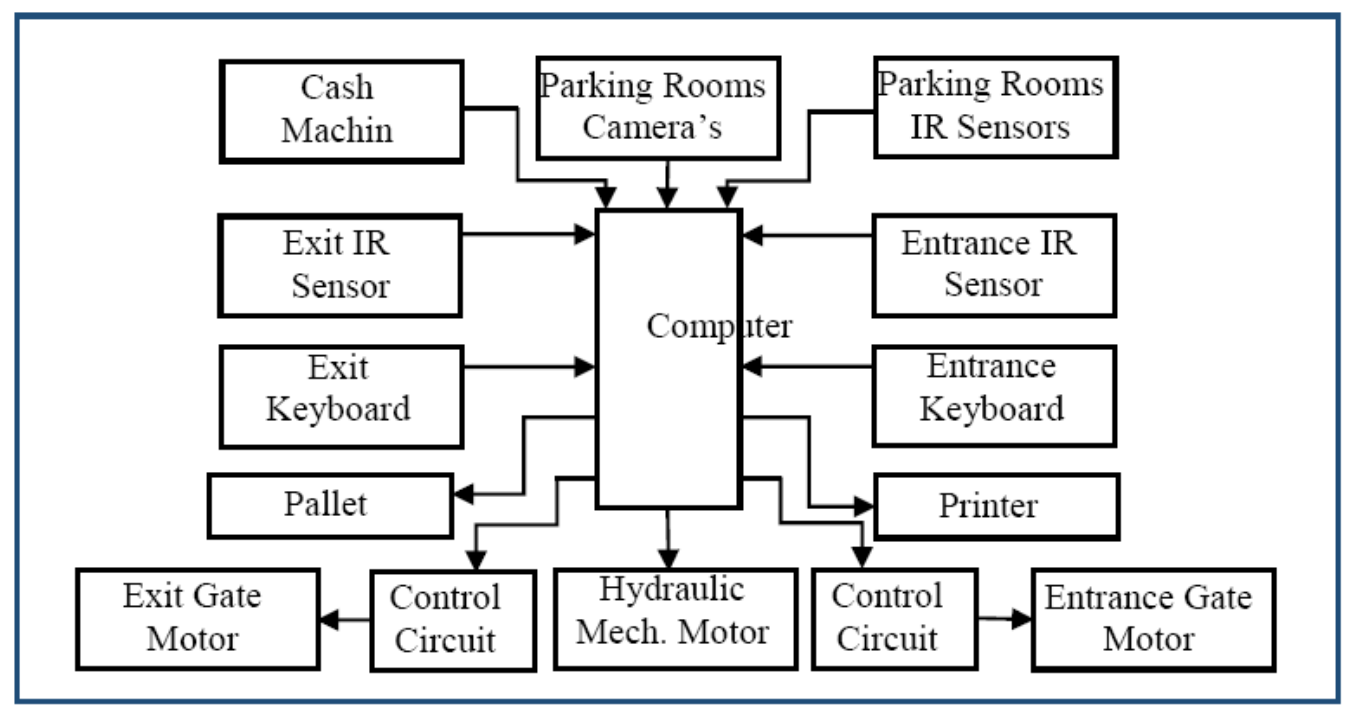

Figure3. Depicts the block diagram of the system

\section{System Software}

The system elements are all performing the different tasks in integrative manner to facilitate the automation concept. In this work, the software is utilized to speed up all the parking process and strengthen the security feature [8]. The software is divided into both parking and retrieving processes. The parking process is also parted into:

1. The payment of parking time period that determined by the car owner.

2. Car parking that carried out automatically by the subroutine of the parking room.

Figures 3 and 4 are flowcharts of car parking and retrieving process which illustrate the sequences of processes and the system integration. The car retrieving is also categorized into:

- Verifying the payment of the additional time period of the parking

- And the subroutine of retrieving car.

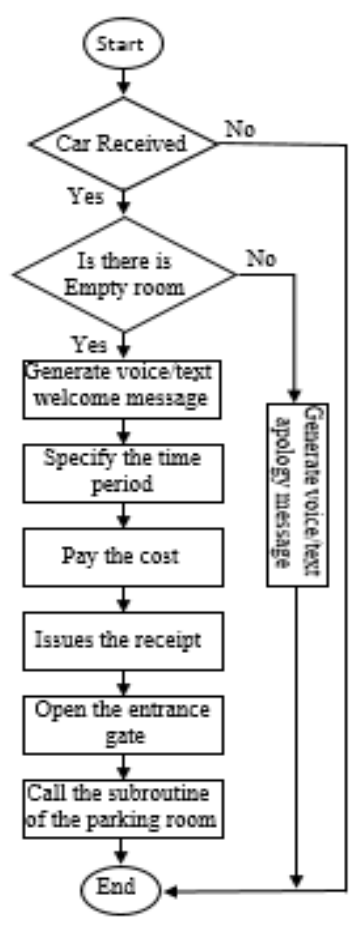

Figure3. Shows the parking process 


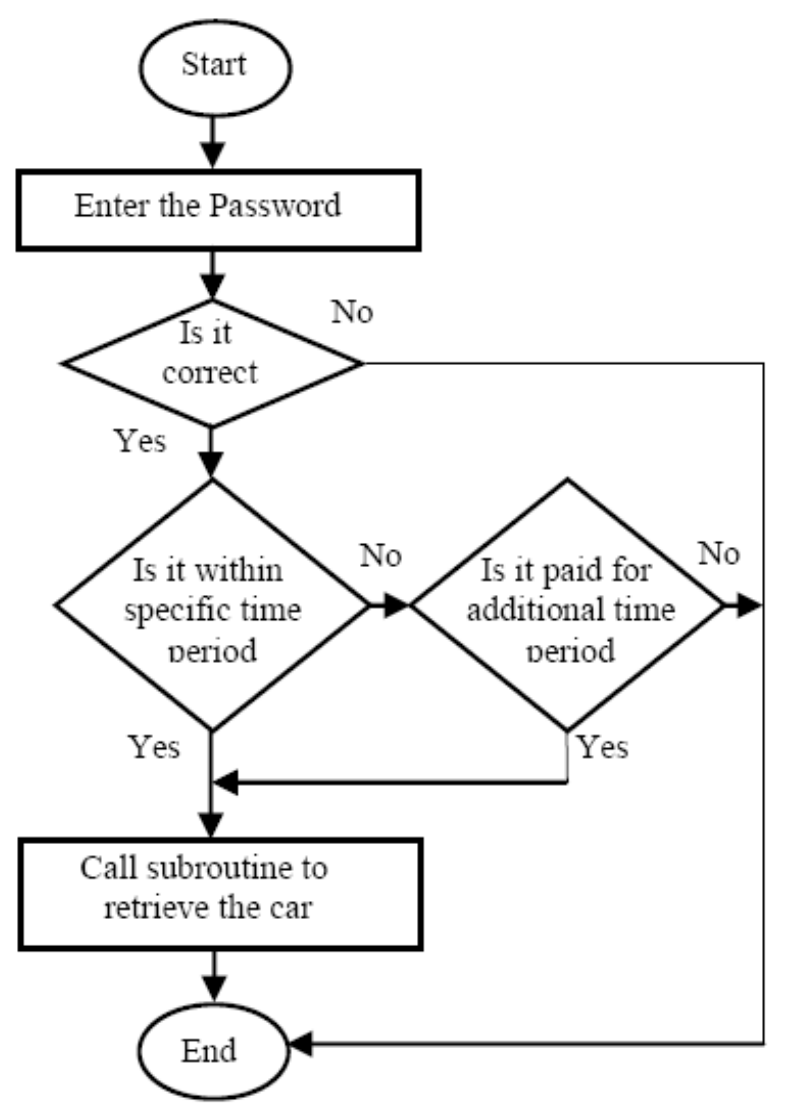

Figure4. Shows the retrieving process

\section{Design And Performance Concept}

The development that made in the designed system may act directly as the assistant tool to help in improving the way of controlling the parking system so as to reduce the total parking area and in same time increasing the total revenue. The sequences of the system scenario are illustrated according to the system vital parts that all together are playing an important role in order to integrate each other for better operation. So, the hardware parts role is defined and clarified as follows:

\subsection{Receiving Room}

Receives the car through entrance gate. The gate checks the system for the empty parking room via sending signal to the system software to find out the nearest empty room(s). If there is/are an empty $\operatorname{room}(\mathrm{s})$, the system software initiates voice/text welcome message and ask the car owner to specify the required period that he want to park the car and its related cost. After the payment, the system issues a printed receipt consisting of paid amount as well as the password for car retrieving. The password contains of parking room and the time of parking process. Then, the car will be delivered to the waiting room where the hydraulic mechanism is found. If there is no empty parking room, the system also initiates voice/text apology message and keep the entrance gate locked and the car out of the system.

\subsection{The Waiting Room}

Contains circular hydraulic mechanism which will not begin to perform the task unless the client steps out from the car which can be checked by exit gate IR sensor. After checking, the exit gate is opened by exit gate IR sensor to let him pass and then closed. the hydraulic mechanism carries out the parking process in order to positioning the car in the right parking room as stated in the receipt. All the sequences of the parking process are performed reliably according to the software program.

\subsection{The Circular Hydraulic Mechanism}

Composed of the lift that has a weight sensor to ensure that the load is related to the car type and not for other object. This mechanism has lift circular movement as well as vertical. In addition, there is also a pallet which has horizontally inward/outward movement. First, the vertical movement stepper motor of the mechanism receives its command from the subroutine of the parking room program to 
move the car to the relevant floor, then the circular movement stepper motor directs the car to the appropriate parking room. Therefore, the pallet stepper motor place the car inside the room as inward movement and back again to its normal. Now, the vertical movement stepper motor also returns to its normal to be ready for serving another client. All the aforementioned processes are monitored by means of camera at the supervisor room for better supervision and control. As the hydraulic mechanism performing all related parking process, both IR entrance and exit gate sensors are blocked and the busy system text/voice message is initiated when there is any object in front of the entrance gate or a client at the exit gate requesting to retrieve his car.

\subsection{Car Retrieving}

When the client requests a system to retrieve the car, the system ask him to enter the password that provided in the receipt to ensure that the client is the only authorized to retrieve his car. The system will check is the time period that the car spent in the parking match to that in receipt. If not, the system requests the client to pay for the extra period or otherwise the system will block the request. If the cost paid, the exit gate will quickly open after the hydraulic mechanism brings the car back via subroutine of car retrieving. In the same time, the entrance gate also opened to enable the car to get out of the system securely.

\section{Conclusion}

The system is designed and simulated for better performance and safety. It introduces some advantages over traditional other system such as flexibility, higher security, higher revenue, small space, and reduce human errors. Because of these advantages, this system can be exploited in hotels, military areas, stadium, shopping malls, railway stations, airports or where there are cars crowding.

\section{ACKNOWLEDGMENT}

Throughout this work, I would like to gratefully thank Mr. Bushra A. Elgabar Salah for spending time in help and support and made his remarks on most aspect of the technical system parts.

\section{REFERENCES}

[1] SwanandS .Vaze, Rohan S. Mithari, Sanjay T. Sanamdikar, "PLC Based Automatic Multistoried Car Parking System", International Journal of Engineering Research and Applications, Vol. 4, Issue 12(Part 6), December 2014, pp(67-71).

[2] Mala Aggarwal, Simmi Aggarwal, R.S.Uppal, "Comparative Implementation of Automatic car Parking System with least Distance Parking Space in Wireless Sensor Network", International Journal of Scientific and ResearchPublications, Volume 2, Issue 10, October 2012.

[3] Ankit Gupta, Ankit Jaiswar, Harsh Agarwal, Chandra Shankar, "Automatic Multilevel Car Parking", International Journal of Electrical and Electronics Research, Vol. 3, Issue 2, Month: April - June 2015, pp: (438-441).

[4] (2002) The IEEE website. [Online]. Available: http://www.ieee.org/

[5] M. Shell. (2002) IEEEtran homepage on CTAN. [Online]. Available:http://www.ctan.org/texarchive/macros/latex/contrib/supported/IEEEtran/

[6] FLEXChip Signal Processor (MC68175/D), Motorola, 1996.

[7] "PDCA12-70 data sheet," Opto Speed SA, Mezzovico, Switzerland.

[8] Elsanosy M. Elamin, Zohair M. Elhassan, "Real Time Automatin dual Gain Control of the Helical feerd Parabolic Reflector Antenna", International Journal of Electrical and Electronic Engineers, IJEEE, Vol.7, Issue 02, July-December 2015, PP(11-16).

\section{AUTHOR'S BIOGRAPHY}

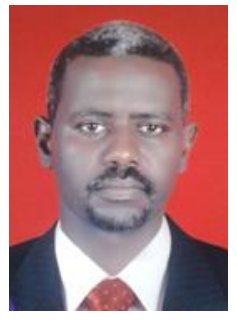

Dr. Elsanosy M. Elamin, Obtained his BSc in Electrical and Computer Engineering at Omdurman Islamin University 1999. Received his MSc. in Communication at Karary Academy of Technology 2003 and Ph.D. in Electronics at Sudan Academy of Sciences 2014. Assistant Professor and head department of electrical engineering, University of Kordofan. His field interest is in Electronic Design, Data Communication Networks, and Mobile Communication Networks. 